sensibilité beaucoup trop faible. Au contraire la plaque à électrons, par exemple la plaque pour recherches nucléaires, Ilford $\mathrm{C}_{2}$, possède des grains très fins et un rendement élevé; elle permet de résoudre partiellement le deuxième problème. Peut-on faire mieux?

Il existe un phénomène atteignant la limite de ces possibilités, c'est le phénomène de 'tenebrescence' des centres $F$ dans les cristaux, en particulier de $\mathrm{KCl}$. D'après $\mathrm{H}$. W. Leverenz(16) le nombre de centres $F$ peut atteindre Io $^{18}$ par $\mathrm{cm}^{3}$; avec un écran de I / Ioo de mm. d'épaisseur, nous aurons par $\mathrm{mm}^{2}{ }^{2}$ d'écran IO $^{\mathbf{1 3}}$ centres récepteurs. Pour des poses de longues durées sur le ciel nocturne, nous restons bien loin de la saturation. D'autre part, les centres $F$ étant à l'échelle atomique, la fluctuation apportée par le récepteur est réduite au minimum. Malheureusement le procédé n'est pas très sensible, mais il est possible d'augmenter l'efficacité de ces récepteurs à structure très fine en diminuant les dimensions de l'image électronique; un grandissement de $\mathrm{I} / 7$ est facilement réalisable. D'autre part l'analyse du signal, c'est-à-dire des densités, pourrait se faire par des méthodes plus sensibles que le simple examen à l'œil, par exemple par des méthodes photo-électriques à balayages.

\title{
BiBLIOGRAPHIE
}

(I) W. A. Baum, Astron. J. 59, 314, 1954 .

(2) Otto Struve, Sky and Telescope, 14, I86 (March) et 224 (April), r955.

(3) A. Lallemand, C.R. 203, 243 et $990,1936$.

(4) A. Lallemand, L'Astronomie, 5I, 300, 1937.

(5) A. Lallemand et M. Duchesne, C.R. 233, 305, I95I.

(6) W. A. Baum, loc. cit.

(7) W. A. Hiltner, Astron. J. 6o, 26, 1955.

(8) P. E. Argyle, Journal of the R.A.S. of Canada, 49, 19, 1955.

(9) A. Lallemand et F. Lenouvel, C.R. 225, $1129,1947$.

M. Bloch et R. Falgon, Publ. de l'Obs. de Lyon, série I, tome V, fasc. Io.

Kaliniak, Krassovsky, et Nikonov, Izvestia de l'Obs. de Crimée, 6, Ir9, I951.

Krassovsky, Rapports à l'Acad. des Sci. de l'URSS, 66, no. I, 1949.

A. Lallemand et M. Duchesne, C.R. 235, 503, 1952.

P. E. Argyle, loc. cit.

(10) V. K. Zworykin et E. G. Ramberg, Photoelectricity and its Application, p. I72 (Wiley, New York, 1949).

(I I) A. Lallemand, C.R. 223, 856, 1946.

(12) $A p . J$. 121, 772, 1955.

(13) Astron. J. 60, I54, 1955 .

(I4) A. Lallemand et M. Duchesne, C.R. 238, 335, I954. Voir aussi Otto Struve, Sky and Telescope 14, 224 (April), 1955.

(I5) A. Lallemand et M. Duchesne, C.R. 240, I329, 1955.

(I6) H. W. Leverenz, R.C.A. Review, 7, r99 (June), I946. Voir aussi Introduction to Luminescence of Solids (Wiley, New York, 1950).

\section{INFORMATION STORAGE AND INTEGRATION APPLIED TO LOW-CONTRAST ASTRONOMICAL IMAGES}

\author{
By G. A. Morton, R.C.A. Laboratories, Princeton, N.J.
}

In observational astronomy one is continually faced with the problem of interpreting faint images which are close to the limit of recognizability. The problem of threshold recognition of images at low light levels may be subdivided into two cases: ( $\mathrm{I}$ ) the limit of detectability of an image against a black background, and (2) the minimum perceptible change of illumination which can be detected at small but finite light levels. Until such time as the much-discussed space platform is launched into its orbit around the 
Earth, the astronomer must be content to view astronomical objects through a thick layer of relatively luminous air. Therefore, the second type of problem, namely that of a low-contrast image, is the main consideration.

In general, an image consists of areas of different surface brightnesses distributed in a pattern over the image plane. The recognition of the image depends upon being able to detect differences in brightness among the various areas. The problem does not differ in kind from the simplest case of detecting the difference in brightness between a single area and its background. Any conclusions as to fundamental limits of detectability in the elementary image can be immediately extended to the general case. Therefore, let us consider the factors involved in recognizing a very simple image formed on the sensitive surface of an image-sensing device which is capable of measuring the radiation density distributed over its surface, e.g. the photosensitive cathode of a television 'pick-up' tube, the retina of an eye, or a photographic plate. It will be assumed to have the property of integrating the information over areas which are equal to its resolution capabilities.

Suppose, for example, that the intensity of illumination within a faint image of area $d^{2}$ is $I_{A}$ while the background intensity surrounding it is $I_{B}$. The contrast between this image and its background is, by definition, $C=\left(I_{A}-I_{B}\right) / I_{B}$. If light were a perfectly continuous medium, the only limit on the smallness of the contrast step which could be detected would be that due to the sensitivity of the device. However, light in its interaction with matter behaves as though it were corpuscular in nature, that is, it behaves as a flux of photons. The arrival of photons at a surface is a purely random phenomenon and is governed by the laws of statistics. If the average rate of arrival of photons upon a particular area is $n$, then the average number of photons expected within an exposure time $t$ is $n t$, but the actual number within any particular intervals $t$ will be subject to a root-mean-square deviation of $(n t)^{\frac{1}{2}}$.

All image-sensing devices of interest in this discussion are based upon the emission of photo-electrons, and the probability that a particular photon will eject a photoelectron is considerably less than unity for any actual photo-emitter. Since the statistics of detection are concerned with the actual number of photo-events utilized rather than with the total number of incident photons, the root-mean-square deviation of interest here is $(q n t)^{\frac{1}{2}}$ where $q$ is the effective quantum efficiency of the image detector. If $\kappa$ represents the number of photons per unit of intensity $I$, the number of registered photo-events within the image will be $q_{\kappa} I_{A} d^{2} t$, while the number of events within an equal background area will be $q_{\kappa} I_{B} d^{2} t$. It is evident that the image cannot be recognized unless the difference between these two quantities is substantially larger than the associated root-mean-square fluctuation. The practical criterion of image recognition can be formulated by introducing a coefficient of certainty $k$, which is the factor by which the image must exceed the root-mean-square fluctuation in order to be accepted as real. For a conservative threshold of image recognition, $k$ might be assigned the value 5 ; the probability of a spurious fluctuation exceeding that margin is extremely small.

Proceeding from these considerations, one can show that the fundamental lower limit of detectable contrast is given approximately by

$$
C \approx k\left(\frac{2}{q \kappa I_{B} d^{2} t}\right)^{\frac{1}{3}}
$$

A more complete discussion of image recognition, limiting stellar magnitude, etc., follows in paper no. 3. For the present, this formula will be sufficient to call attention to the important role played by the integration time $t$. Under a given set of conditions, the threshold contrast required for image detectability decreases when the integration time increases. The information integration time is sometimes a variable which is at the disposal of the observer and which furnishes a valuable means of increasing contrast discrimination. The remainder of the present paper is devoted to a consideration of electronic techniques for incorporating information integration into a photo-electric image-receiving system. 
The first electronic image-receiving device employing the integration principle to enhance its sensitivity was the iconoscope. It was followed by the orthicon, which incorporated the same integration principle at its light-sensitive target but which differed in the way that the scanning beam was used for reading the information stored on the target. A further increase in sensitivity was achieved with the development of the image orthicon. The image orthicon and its various first cousins are the 'pick-up' tubes most widely used by the television industry at the present time.

The construction of the image orthicon is shown diagrammatically in Fig. 2. The primary sensitive surface is a photocathode on the glass end of the tube. The storage target is mounted about $2.5 \mathrm{~cm}$. away from the cathode and takes the form of a thin glass sheet having a resistivity between ${ }^{10}{ }^{10}$ and ${ }^{10} 0^{12} \mathrm{ohm}-\mathrm{cms}$. The glass is stretched tightly on a metal supporting ring, and a secondary electron collector grid, spaced about $0.1 \mathrm{~mm}$. from the target on the cathode side, is mounted on the same frame. The electron

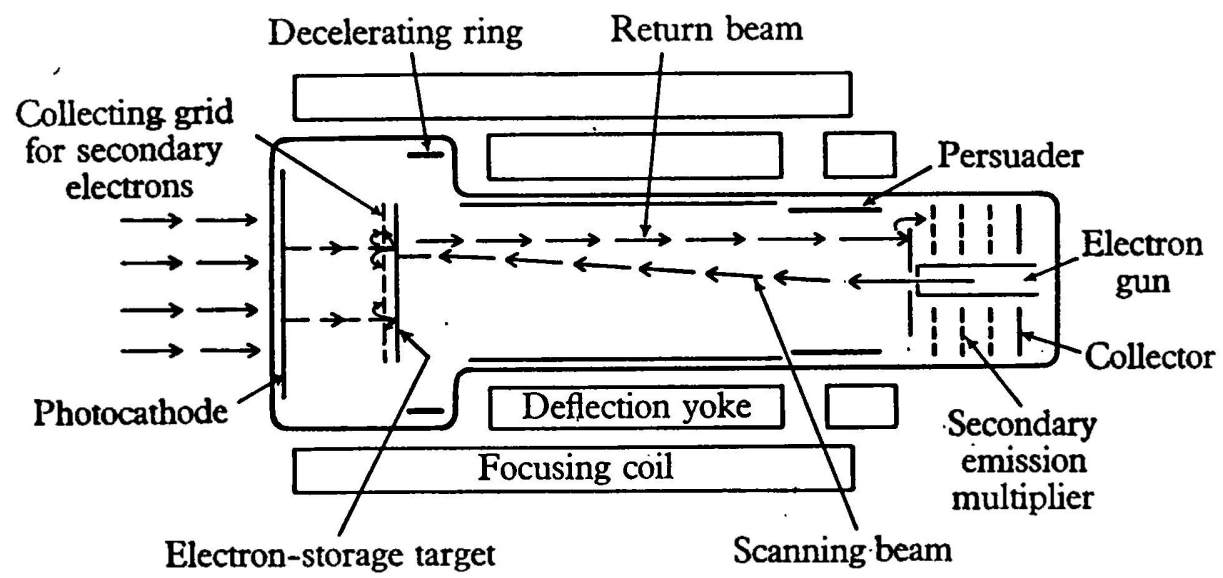

Fig. 2. Schematic diagram of an image orthicon. Light incident from the left ejects electrons from the photocathode. These, in turn, eject secondary electrons from the target. This electrically stored image on the target is 'read' periodically by scanning it with a beam of electrons whose deflection due to the charges on the target is detected and amplified.

gun which produces the scanning beam is located at the opposite end of the tube, and surrounding it is a secondary emission multiplier. The electrons from the gun are focused into a narrow beam by a magnetic focusing coil which surrounds the tube, and are deflected by magnetic deflecting coils so that the electron spot scans the target in a series of straight parallel lines. The beam electrons strike the target with very low velocities so that they produce almost no secondary electrons.

When no light strikes the photocathode and the target is scanned by the beam, the target surface becomes increasingly negative due to the electrons it accumulates. As soon as the target has been driven to a potential slightly below that of the gun, the beam electrons can no longer reach the target and are turned back. The returning electrons are collected and amplified by the secondary emission multiplier.

The optical image being detected is focused onto the photocathode, causing electrons to be emitted in a distribution pattern or electron image which corresponds to the light image. The electron image is focused onto the target, and the potentials are so arranged that the electrons strike it with a velocity corresponding to 300 volts. At this velocity, each electron produces three or four secondary electrons. It should also be mentioned that separating the photocathode from the target has the added advantage that the electron image can be deflected and gated.

Fixing our attention on one picture element of the target, we note that this element is driven to equilibrium (gun potential) as the scanning beam passes over it. As the scanning beam moves away, the element is charged increasingly positive by the secondary 
emission produced by the image electrons. This positive charge accumulates (integrates) all during the time that the beam is away. When the beam returns, it again drives the element to equilibrium. When the beam passes over an element which has accumulated a positive charge, fewer electrons return to the multiplier than when the element is not charged. Thus the output current of the multiplier is low when the beam passes over elements corresponding to lighted areas of the photocathode and is greater when the beam passes over elements corresponding to dark areas. This constitutes the video signal output. After amplification, this signal can be supplied to the grid of a kinescope (a viewing tube such as the picture tube of a television receiver) whose scanning beam is synchronized with that of the image orthicon. The electronically accumulated image is thereby reproduced where it can be viewed and recorded photographically.

The important point to notice in connexion with the operation of the image orthicon is that the photo-electric information is integrated on the target during the time it takes the beam to traverse the target. As ordinarily used in television, the scanning period is $\mathrm{r} / 30$ sec. However, if the target insulation were high, the charge could be integrated

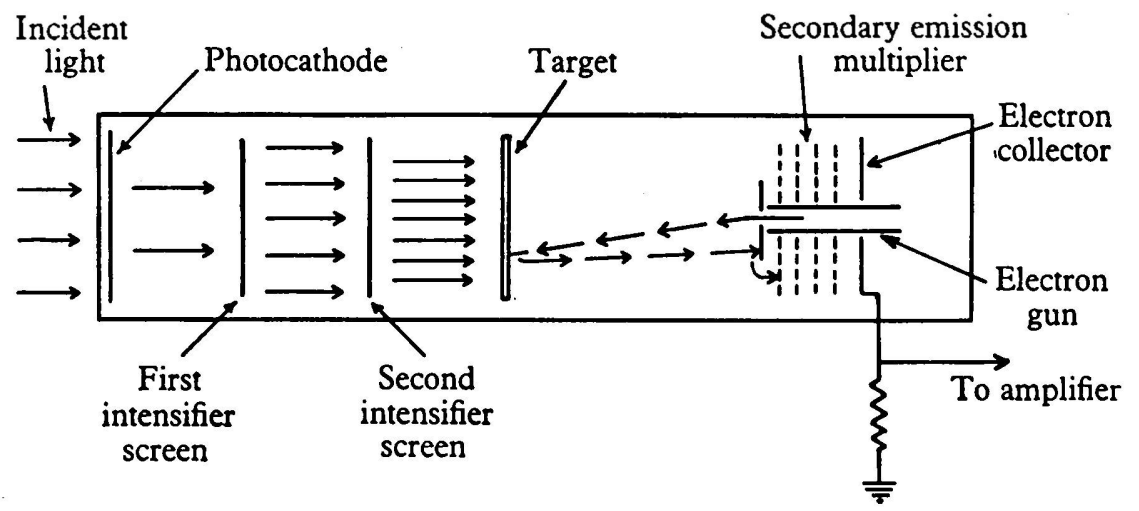

Fig. 3. Schematic diagram of an intensifier orthicon. Between the photocathode and the target (see Fig. 2) are two intensifying screens. The number of electrons coming out of each screen is about ten times the number of electrons incident upon it from the left. The image is preserved by refocusing it upon each screen.

over periods of many minutes. While the image orthicon is very sensitive, it does not reach the fundamental contrast threshold specified by the formula. The statistical fluctuations which limit image recognition are those due to the 'shot noise' of the scanning beam, rather than the statistical fluctuations of the primary photo-electrons.

In order that each photo-electron may cause the accumulation of enough charge on the target so that the principal fluctuations in the video signal are due to the statistics of photo-electrons rather than beam noise, the primary photo-electron image current must be amplified by a factor of 200 or 300 before it reaches the target. Fig. 3 is a schematic diagram of a tube incorporating such an amplifier or intensifier. Tubes embodying this intensifier principle have been investigated in connexion with making faint X-ray images more easily visible on fluoroscopic screens. These intensifier orthicons (Fig. 3) employ a primary photocathode in the form of a semi-transparent film on the glass end of the tube. The electrons from this cathode are focused by means of an electrostatic lens system onto an intensifier screen. This intensifier screen consists of a thin glass membrane coated on the image side with an aluminium-backed layer of fluorescent material (a phosphor) and on the other side with a photocathode whose spectral response matches that of the phosphor. Each impinging electron produces a shower of photons in the phosphor, and these photons in turn eject a shower of new photo-electrons from the photocathode side of the membrane. With an accelerating potential of 10,000 volts between the primary photocathode and the first intensifier screen, each primary electron causes the emission of ten electrons from the screen. The electron image from the first 
intensifier screen is focused onto a second similar intensifier screen which also has an amplification factor of ro. The electrons from this second intensifier screen are imaged onto a storage target identical with that used in an ordinary image orthicon. Therefore, every photo-electron from the primary cathode results in the emission of approximately 300 electrons from the target. This amplification is sufficient for the additional spurious noise from the scanning beam to be small compared with the fundamental statistical noise due to the primary photo-electrons.

The sensitivity of experimental tubes of this type built for X-ray studies has been found to be at least two orders of magnitude higher than that of ordinary image orthicons. Their threshold contrast detection is essentially that given by the fundamental formula. With these tubes, an image whose definition is limited to 400 lines can be obtained with a cathode illumination of the order of $\mathrm{IO}^{-6}$ or $\mathrm{IO}^{-7}$ foot-candles.

These experimental intensifier orthicons are capable of much longer storage times than the I/30 sec. which is normally used. Without any modification of the tube design, but with the use of special circuitry, integration times of several minutes have been obtained. By the use of specially processed targets having much higher insulation than is normally used, electrical storage up to an hour or more could probably be achieved. This estimate is based on results already obtained with storage orthicons.

An interesting possibility arises if the gain between the photocathode and the target of an intensifier orthicon could be made sufficiently high. If each photo-electron results in enough charge being stored on the target to stand out above instrument noise, it can be reproduced on a kinescope as an individually observable scintillation. The kinescope would be biased so that it is completely black except at each scintillation. The television chain would be operated at the normal frame rate of 30 cycles per sec. and a conventional camera would photograph the results. Under these circumstances, each photo-electron would yield a recorded event, and the results should be fundamentally similar to those obtained with an image converter incorporating a photographic plate as described by Prof. Lallemand in paper no. I. Such an arrangement would preserve all of the information contained in the primary photo-electron flux without introducing additional spurious signals and without placing the entire burden of information integration on the tube itself. It would not, however, permit the night-sky background to be subtracted away in the manner that it can when an ordinary image orthicon is used.

Each of the two methods of obtaining integrated image information described above, namely, charge integration on the target and photographic integration of reproduced scintillations, should lead to pictures whose contrast information is limited only by the photo-electric process of the cathode. This is true only, however, as long as the storage capabilities of the target or the photographic plate are not exceeded. Frequently, in astronomical work, the image being observed has an extremely low contrast against a relatively bright background. Under these circumstances, the information storage capacity of the system may be the real limiting factor. For the photographic plate this limit is reached when the exposed grain density becomes large enough for a significant fraction of the grains to hide behind one another and no longer contribute useful information (see paper no. 3). For an image orthicon, if the accumulated positive charge on the target produces a potential of more than one or two volts, the potential of the secondary electron collector grid is exceeded, and the incident image electrons no longer produce secondary emission as efficiently.

Let us now consider how resolution affects information storage. Suppose, for example, that the electron optics and the target of an image orthicon were capable of extremely high resolution so that each photo-electron would yield a discrete bundle of charge (on the target) which would not touch or blend into neighbouring charges. Specifically, we suppose here that these charged points are small compared with the disk over which a faint star image is smeared by atmospheric 'seeing'. If this array of charged points were scanned with a very sharp reading beam which encountered the charges one at a time, the output signal of the image orthicon would be a series of individual pulses and it would be reproduced as discrete scintillations on the kinescope. If a bias were imposed 
on this signal in an effort to lower the kinescope background, all of the scintillations would decrease equally in brightness both within the area occupied by a star image and in the background surrounding it. Under these circumstances, none of the sky background could be subtracted without weakening the wanted images to the same degree. In other words, the contrast could not be enhanced at all if perfect resolution were preserved throughout the system. As a result, the information storage capacity of the system would need to accommodate all of the sky background in addition to the images superposed upon it.

If, on the other hand, the charge resulting from each photo-electron is spread over a sufficient area to blend considerably into neighbouring charges, the output signal of the image orthicon will consist of a continuous background with small fluctuations. Under these circumstances, most of the background can be subtracted before the final picture is recorded, say, by photographing the kinescope. This subtraction trick provides an opportunity for increasing the integration capacity of the system, because most of the sky background does not need to be stored after the signal is removed from the target.

One can therefore envisage a two-step process of image information integration. The image orthicon is defocused to a degree where its resolution is equal to the minimum detail to be preserved in the picture. The target is scanned at intervals of every few minutes so that appreciable charge integration is obtained, but the storage capacity of the target is not exceeded. The output signal is biased to a degree where most of the background is discarded and only the residual portion containing the statistical fluctuations is delivered to the screen of the kinescope. These background-clipped pictures which are flashed periodically on the kinescope can be integrated photographically in one long exposure until the storage capacity of the photographic plate is used up.

It is difficult to calculate theoretically the gain in information storage that is achieved by this two-step procedure, because various non-fundamental effects would undoubtedly be encountered in a system so complex as the one just outlined. However, two or three orders of magnitude in storage capacity might be obtainable by this technique. Almost no experimental work of this nature has been done, and there is little point in trying to estimate possible performance more accurately until tests can be made.

An effort has been made in this paper to point out some of the ways in which present television techniques and their logical extensions may be of value in the field of astronomy. It is evident that much experimental and developmental work is needed in this field. For example, a tube could be designed which would filter out the unwanted uniform background information and thus conserve valuable storage capacity. Such a tube would make the two-step process outlined above unnecessary, yet it would accomplish the same result. This tube could be designed and operated in such a way that in addition to giving background filtering it would also give continuous presentation of the stored information. The development of this and other tubes which might be of considerable astronomical value can come only as the result of adequate theoretical and experimental research.

\section{THE DETECTION OF FAINT IMAGES AGAINST THE SKY BACKGROUND}

\section{By W. A. BAum, Mount Wilson and Palomar Observatories}

The detection of fainter and fainter objects in the sky has been of fundamental importance to modern advances in observational astronomy and cosmology, and it has led to the building of bigger and bigger telescopes in an effort to collect more and more light. A point has now been reached where the cost of building telescopes appreciably bigger than those now in existence becomes prohibitive, but the perfection of better image receivers for the light already available stands a good chance of accomplishing the same thing at comparatively modest expense. 